undulation, reflecting a rhythm and possibly significant of a remote pendulum whose rate of vibration is known. If it can be traced to such a pendulum there will result a determination of the rate at which the chronograph scroll moved when that part of the record was made : and a moderate number of such determinations, if well distributed, will convert the whole scroll nto a definite time scale.

In other words, if a sufficient number of the rhythms embodied in strata can be identified with particular imposed rhythms, the rates of sedimentation under different circumstances and at different times will become known, and eventually so many parts of geologic time will have become subject to direct calculation that the intervals can be rationally bridged over by the aid of time ratios.

For this purpose there is only one of the imposed rhythms of practical value, namely, the precessional ; but that one is, in my judgment, of high value. The tidal rhythm cannot be expected to characterise any thick formation. The annual is liable to confusion with a variety of original rhythms, especially those connected with storms. The rhythm of eccentricity, being theoretically expressed only as an accentuation of the precessional, cannot ordinarily be distinguished from it. But none of these qualifications apply to the precessional. It is not liable to confusion with the tidal and annual because its period is so much longer. being more than 2000 times that of the annual. It has an eminently practical and convenient magnitude, in that its physical manifestation is well above the microscopic plane, and yet not so large as to prevent the frequent bringing of several examples into a single view. It is also practically regular in period, rarely deviating from the average length by more than the tenth part.

From the greater number of original rhythms it is distinguished, just as from the annual and tidal, by magnitude. The practica geologist would never confuse the deposit occasioned by a single storm, for example, with the sediments accumulated during an astronomical cycle of 20,000 years. But there are other original rhythms, known or surmised, which might have magnitudes of the same general order, and to discriminate the precessional from these it is necessary to employ other characters. Such characters are found in its regularity or evenness of period, and in its practical perpetuity. The diversion of the mouth of a great river, such as the Hoang Ho or the Mississippi, might recur only after long intervals; but from what we know of the behaviour of smaller streams we may be sure that such events would be very irregular in time as well as in other ways. The intervals between volcanic eruptions at a particular vent or in a particular district may at times amount to thousands of years, but their irregularity is a characteristic feature. The same is true of the recurrent uplifts by which mountains grow, so far as we may judge them by the related phenomena of earthquakes and the same category would seem to hold also the theoretically recurrent collapse of the globe under the strains arising from the slowing of rotation. The carbon dioxide rhythm, known as yet only in the field of hypothesis, is hypothetically a running-down oscillation, like the lessening sway of the cradle when the push is no longer given.

But the precessional motion pulses steadily on through the ages like the swing of a frictionless pendulum. Its throb may or may not be caught by the geological process which obtains in a particular province and in a particular era, but whenever the conditions are favourable and the connection is made, the record should reflect the persistence and the regularity of the inciting rhythm.

The search of the rocks for records of the ticks of the precessional clock is an out-of-door work. Pursued as a close study it could have no satisfactory outcome, because the printed descriptions of rock sequences are not sufficientiy complete for the purpose ; and the closet study of geology is peculiarly exposed to the perils of hobby-riding. A student of the time problem cannot be sure of a persistent, equable sedimentary rhythm without direct observation of the characters of the repeated layers. He needs to avail himself of every opportunity to study the series in its horizontal extent, and he should view the local problem of original versus imposed rhythm with the aid of all the light which the field evidence can cast on the conditions of sedimentation.

Neither do I think of rhythm seeking as a pursuit to absorb the whole time and energy of an individual and be followed steadily to a conclusion; but hope rather that it may receive the incidental and occasional attention of many of my colleagues

No. 1603 , VOL., 62$]$ of the hammer, as other errands lead them among cliffs of bedded rocks. If my suggestion should succeed in adding a working hypothesis or point of view to the equipment of field geologists, I should feel that the search had been begun in the most promising and advantageous manner. For not only would the subject of rhythms and their interpretations be advanced by reactions from multifarious individual experiences, but the stimulus of another hypothesis would lead to the discovery of unexpected meanings in stratigraphic detail.

It is one of the fortunate qualities of scientific research that its incidental and unanticipated results are not unfrequently of equal or even greater value than those directly sought. Indeed, if it were not so there would be no utilitarian harvest from the cultivation of the field of pure science.

In advocating the adoption of a new point of view from which to peer into the mysterious past, I would not be understood to advise the abandonment of old standpoints, but rather to emitlate the surveyor, who makes measurement to inaccessible points by means of bearings from different sides. Every independent bearing on the earth's beginning is a check on other bearings, and it is through the study of discrepancies that we are to discover the refractions by which our lines of sight are warped and twisted. The three principal lines we have now projected into the abyss of time miss one another altogether, so that there is no point of intersection. If any one of them is straight, both the others are hopelessly crooked. If we would succeed we should not only take new bearings from each discovered point of vantage, but strive in every way to discover the sources of error in the bearings we have already attempted.

\section{THE RELATION OF STIMULUS TO}

\section{SENSATION.}

NOTHING has done more to place on a scientific footing the discussion of the phenomena which the study of matter and energy presents to the eye of reason, than the establishmeut of a doctrine of quantitative equivalence. So much oxygen and hydrogen, so much water; this amount of energy of chemical separation gone, that amount of sensible heat gained. In a similar way, nothing is likely to do more to give support to the hypothesis that sentience or consciousness is a concomitant of certain physiological processes than the establishment of a quantitative relation between stimulus and sensation.

It has, indeed, long been obvious that some general relation of this kind holds good. Increased physical pressure is, within certain limits, increasingly felt; more light gives a higher degree of visual sensation; the greater the amplitude of the vibrations of a violin-string the fuller and louder the sound. Such statements are, however, indefinite. We want to know how much the physical increase must be to give just so much increment in sensation. If we double the strength of the stimulus, do we double also the strength of the sensation? If not, by how much do we increase it? Ernst Heinrich Weber sought to express the quantitative relation with some exactness; Gustav Theodor Fechner and other more recent inquirers have built upon the foundations laid by Weber; and a provisional law of the relation of physical stimulus to felt sensation has gradually gained wide acceptance.

Weber's classical experiments dealt with what is termed the "least observable difference." If, for example, a weight of one pound be laid upon the hand, it gives rise to a sensation of pressure. If, now, an extra ounce be added no difference is felt, nor is the added weight of two or of inree ounces perceptible. The sensation is not increased, and then only just perceivably increased, till one-third of a pound is added. This, then, is said to be the least observable difference. We now start afresh with a load of two pounds, and add, as before, one-third of a pound. But there is no observable difference; nor is there any felt increase in sensation until two-thirds of a pound are added. Starting once more with an initial load of three pounds, we find that neither the addition of one-third, nor that of two-thirds of a pound affords any observable difference in the sensation experienced. A full pound must be added for the increment to be felt. The least observable differences, therefore, are between

$$
\begin{aligned}
& \text { I lb. and } I+\frac{1}{3} \mathrm{lb} \text {. } \\
& 2 \mathrm{lb} ., 22+3 \mathrm{l} \text {. } \\
& 3 \mathrm{lb},, \quad 3+\frac{3}{3} \mathrm{lb} \text {. }
\end{aligned}
$$

If, then, we extend and generalise the results of such experiments, we find that, within certain limits, to obtain an orderly 
series of just observable differences in sensation we must always add the same fraction - one-third of the weight - at each constant step of the series.

Now Fechner assumed that these just perceivable increments of sensation are all of the same value, or are constant; in which case they form an arithmetical series-that is to say, one that is produced by successive additions of the same amount. But the corresponding series of stimuli are not in arithmetical progression, since the successive increments are not of the same amount. The increase is, however, always by the same proportional amount. Each successive stimulus has to be multiplied by a constant factor, $\frac{4}{3}$. The series, therefore, forms an orderly sequence in geometrical progression.

We thus reach what is known as the Weber-Fechner formula, by which the relation of stimulus to sensation is expressed in quantitative terms. It may be thus stated:-To obtain an arithmetical series of sensations a geometrical series of stimuli is required. To give the former, equal increments of sensation are added; to obtain the latter we must muitiply the successive stimuli by a constant factor.

It must be admitted, however, that the results of a great number of carefully-conducted observations are by no means in satisfactory accordance with this formula. Hering and his pupils have shown that for very small stimuli, lying near the threshold of sensation, both stimulus and sensation increase very nearly pari passu in arithmetical progression. The WeberFechner formula cannot, therefore, at present be regarded as more than an approximation to the truth.

In extracting the Weber-Fechner formula from the data afforded by observations on the method of least observable difference, it is necessary to piece together the results observed singly and in succession. But from the nature of the field of vision it is possible to obtain a series of increments of stimulus which shall afford a scale of sensation visible as a whole and at a glance. In the current number of the Psychological Review (vol. vii. No. 3, p. 217 ) I have published in detail the results of an investigation "On the relation of stimulus to sensation in visual impressions," by which I have been led to suggest a modification of the Weber-Fechner formula. Stripped as far as possible of technicalities, the method and results may be here briefly described.

It is well known that if a disc with white and black sectors be rapidly rotated, the effect on the eye is a uniform grey. If the white sectors are proportionally small, occupying, for example, only 5 per cent. of the disc, the effect is that of a very dark grey; if they are relatively large, occupying, say, 90 per cent. of the disc, the effect is that of a very light grey. With such sectors the same proportional amount of white is introduced in all parts of the disc, so as to give in each case the same shade of grey throughout its whole extent. But it is possible to introduce varying proportions of white from centre to circum. ference, and when this is done the rotating disc no longer presents all over its surface the same uniform shade of grey, but shows varying shades. Let us now endeavour to reduce these varying shades to order. Let us arrange the proportions of white stimulus which we introduce, in such a way as to leave a ring of full black (with no white) at the circumference, and to give a ring of full white (with no black) near the centre, and between these extremes to obtain a perfectly smooth and even gradation of shades of grey from one so dark as to be scarcely distinguishable from black, to one so light as to be scarcely distinguishable from white. We may then, when the disc is rapidly rotating, run our eye from white near the centre, through deepening and deepening grey, to black at the circumference, with nowhere any observable jump in sensationnowhere, so to speak, a steeper slope of change than elsewhere ; as if, in fact, we were passing along a perfectly even inclined plane of sensation from the lowest depth of black to the extreme height of white. If we succeed in this-and it is by no means easy of attainment-we shall have secured an arithmetical series of sensation. From one end to the other we have at successively equal distances constant increments of white sensation, just as in ascending a uniform incline we gain equal increments of height for every yard we progress towards our goal. This even slope of sensation is produced by the juxtaposition of all the least observable differences whose sum gives the full scale. Having obtained this result we are able to ascertain, by careful angular measurements of the proportional areas of white at different parts of our disc, the exast amounts of stimulus which are affecting the eye from these several parts. We may, for example, subdivide the area of the disc lying between the inner white circle and the outer ring of black, by drawing nine concentric circles equidistant from each other, and at these nine distances make angular measurements of the proportional amounts of white to black; and then, by plotting, sweep a curve of stimulus through points representing these measured amounts.

When these amounts are tabulated and dealt with by appropriate mathematical methods, it is found that they are not in accordance with the Weber-Fechner formula. Nor does a disc prepared in accordance with this formula give the smooth and evenly-graded incline of an arithmetical series in sensation. For details the reader may be referred to the paper in which the observations and calculations are set forth. The accompanying figure gives the results plotted in a curve on the graphic method The dotted steps indicate the nine measured increments. The vertical distance of any point on the curve, measured from below, upwards, gives the percentage of sensation. The horizontal distance, measured from left to right, gives the corresponding percentage of white stimulus. The law which results from a discussion of these observations, and of others where red,

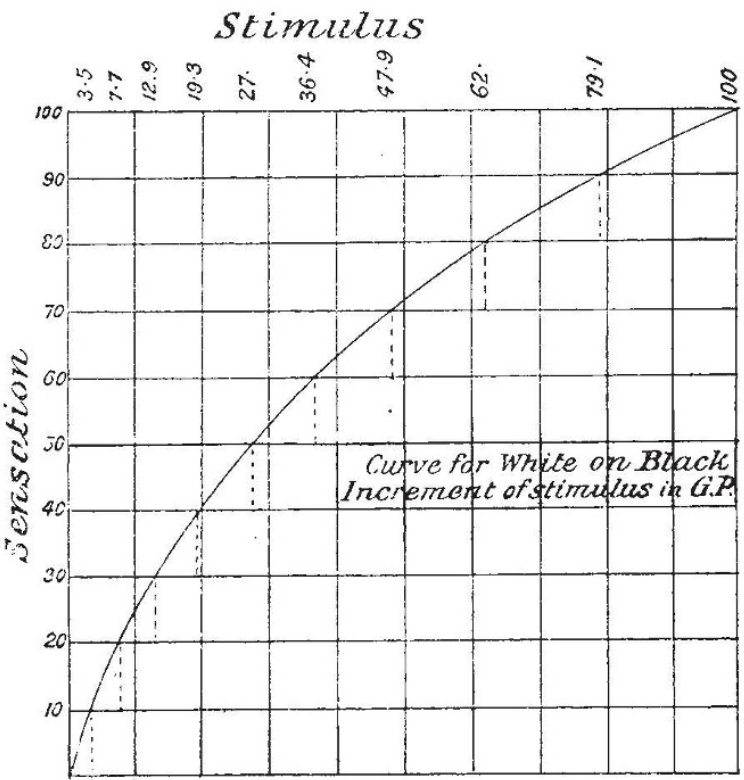

orange and blue stimuli were used instead of white (each of which gives a different curve on the same principle), may be thus formulated :- For constant increments of sensation the concomitant increments of stimulus are in geometrical progression. This differs from the Weber-Fechner formula in assigning the geometrical progression to the successive increinents of stimulus.

The subjoined table gives the increments and sums of White on Black.

\begin{tabular}{|c|c|c|c|c|c|}
\hline \multirow{2}{*}{ Stage } & \multicolumn{2}{|c|}{ Sensation } & \multicolumn{2}{|c|}{ Stimulus } & \multirow{2}{*}{$\begin{array}{l}\text { Observed } \\
\text { percentage } \\
\text { of stimulus }\end{array}$} \\
\hline & Increments & Sums & Increments & Sums & \\
\hline IO & 10 & 100 & 20.90 & 100 & 100 \\
\hline 9 & Io & 90 & $17 \cdot 13$ & 79.10 & 79 \\
\hline 8 & ro & 80 & 14.03 & 6197 & 62 \\
\hline 7 & ro & 70 & II. $5 \mathrm{I}$ & 4794 & 48 \\
\hline 6 & Io & 60 & $9 \cdot 43$ & 36.43 & $35^{\circ} 8$ \\
\hline 5 & Io & 50 & 773 & 27 & 27 \\
\hline 4 & ro & 40 & $6 \cdot 33$ & 19.27 & 19.5 \\
\hline 3 & IO & 30 & 5.20 & I 2.94 & 13 \\
\hline 2 & I0 & 20 & 4.25 & $7 \cdot 74$ & 7.9 \\
\hline 1 & ro & IO & 3.49 & 349 & 3.5 \\
\hline 0 & 0 & 0 & 0 & 0 & 0 \\
\hline
\end{tabular}

No. 1603 , vOL. 62$]$ 
stimulus and sensation at ten stages between black and white. A comparison of the last two columns will show the extent of agreement between observation and calculation. The numbers given under the head of stimulus are calculated on the basis of the suggested law, the number 27 per cent. of stimulus, as the concomitant of 50 per cent. of sensation, being taken over from observation as a basis for calculation.

Although I venture to hope that the results of this investiga. tion contribute something towards a solution of the problem, still it will be seen that we have as yet by no means reached the stage at which we can claim that a law expressing the quantitative relation of stimulus to sensation is established beyond question. But from the work of many observers we may at least draw the conclusion that there is some well-defined relation, though its law at present eludes the grasp of our generalisation. And this so far lends support to the doctrine of concomitance.

There has been much discussion as to the true meaning of the relation. Is it primarily a relation becween physical stimulus and physiological response, or between physiological response and psychological concomitant? In other words, is the law we seek primarily a physiological or a psychological law? We were only $\mathrm{I} \cdot 22$ inch in diameter, and the length of the arms, from the centre of cup to the spindle, only 1.96 inch. The author describes at length the whirling apparatus used in making the experiments, and which had been previously used in the year 1888 , but in an enclosed space, instead of in the free air. $\mathrm{He}$ points out that a whirling apparatus is absolutely necessary for testing anemometers, because we have no other means of accurately measuring the speed of the wind to which the instrument is exposed, unless we employ for that purpose some other anemometer, which must itself be first tested. In the author's view, the effect of using the whirler in the open air is to alternately add to and subtract from the artificial wind resulting from the steady motion of the whirler, so that the actual resultant wind affecting the anemometer acquires a gusty character which is analogous to the conditions always existing in the free air, and the artificial gusty wind thus secured affords a highly appropriate test-wind for anemometers that are to be used in the open air. The apparatus employed is shown in a plate, which we reproduce.

The arm, on the extreme end of which the anemometer $\cdot$ is placed, is 28 feet long, and is made to rotate either by handpower or by means of the engine used in the kite experiments.

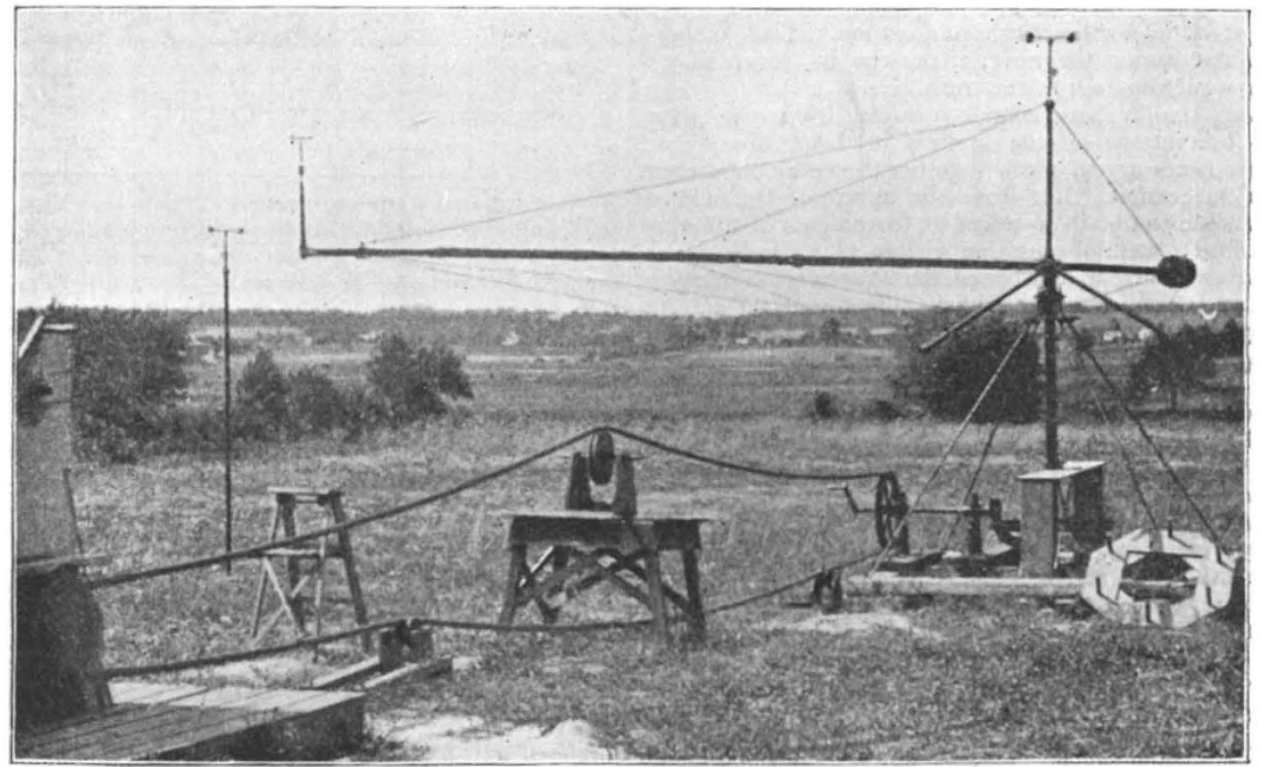

FIG. r. - Whirling Machine and Driving Belt for Anemometer Tests.

cannot enter upon the discussion here. Attention may, however, be drawn to two facts:-First, that Prof. Pfeffer claims to have shown that the attractive influence of malic acid on the spermatozoids of ferns is approximately in accordance with the Weber-Fechner formula; and secondly, that Dr. Augustus Waller's researches on the excitation of muscle and nerve indicate some such relation, though not exactly this relation, between stimulus and physiological response. In view of these facts it seems not improbable, therefore, that the relation may prove to be primarily physiological. In which case we may infer that sensation is directly proportional to the molecular disturbance in the nerve-centres $c^{\wedge} n$ cerned.

\section{Lloyd Morgan.}

\section{ANEMOMETER TESTS.}

THE U.S. Monthly Weather Review for February contain an important contribution by Prof. C. F. Marvin on anemometer tests. The paper gives the results of a series of experiments to determine the factor of an anemometer specially designed for use with kites at considerable altitudes in the free air. For that purpose the anemometer has necessarily to be very small and light, and in the present case the cups
By hand-power any speed up to thirty-five miles an hour could be obtained, and by the engine the velocity could be raised to nearly sixty miles an hour. A good break-circuit seconds pendulum clock was employed, in conjunction with an astronomical chronograph, to record results, and the series of comparisons appears to have been carried out with much care and completeness.

The experiments included a redetermination of the constants for a "standard aluminium cup anemometer," in which the cups were 4.07 inches in diameter, and the arms 6.65 inches in length. This instrument had been used in the investigations of 1888 , and the values now obtained gave a slightly lower rate of speed of the cups in a given wind than had been formerly deduced. But as the differences did not exceed 2 per cent., it is fair to conclude that, upon the whole, the agreement was satisfactory.

The author also points out that another result of the experiments is to confirm a conclusion arrived at in 1888, viz. that an anemometer with large cups, as compared with the length of the arms, runs at a speed bearing a more nearly constant ratio to that of the wind than an anemometer with relatively larger arms. In the case of the small kite anemometer now investigated, the factor is practically constant for velocities from ten to fifty miles an hour, the extreme variation being only about $I$ ' 5 per cent. NO. I603, VOL. 62] 